\title{
Pricing the ecosystem and taxing ecosystem services: a general equilibrium approach
}

\author{
Thomas Eichner and Rüdiger Pethig \\ University of Siegen \\ Department of Economics \\ Hoelderlinstr. 3 \\ D-57068 Siegen \\ Germany \\ phone: ++49 (0) $2717403164 / 3143$ \\ fax: ++49 (0) 2717402678 \\ e-mail: eichner@vwl.wiwi.uni-siegen.de \\ e-mail: pethig@vwl.wiwi.uni-siegen.de
}

\begin{abstract}
In an integrated dynamic general equilibrium model of the economy and the ecosystem humans and wildlife species compete for land and prey biomass. We introduce a competitive allocation mechanism in both submodels such that economic prices and ecosystem prices guide the allocation in the economy and in the ecosystem, respectively. We distinguish the scenarios of an open accessible habitat and a privately owned habitat. In both scenarios efficiency requires different corrective taxes/subsidies to internalize consumption services externalities. In the case of an open access habitat additional sources of inefficiency are the divergence of prices for biomass and land in both subsystems. Finally, we determine values of all components of the ecosystem in an efficient steady state with special emphasis on the role and the interplay of ecosystem and economic prices.
\end{abstract}

JEL classification: H21, Q28

Key words: land, biomass, ecosystem services 


\section{The problem}

Despite our rich knowledge of significant and large-scale interdependencies between the ecosystem and the economy (Alcamo et al., 2003), many pertaining environmental-economic studies tend to be somewhat unbalanced by offering an elaborate analysis of economic activities and far less elaborate modeling of the ecosystem and ecological feedback effects. To the extent that such studies "... do merge economic and ecosystem concepts [they] tend to address isolated markets and a very few species" (Tschirhart, 2000, p. 13). Such approaches offer limited insights only in the complex impacts on the ecosystem of human activities such as land conversion for economic use or biomass harvesting. These economic activities have ramifications and trigger adaptations in the ecosystem and eventually adversely affect ecosystem services that support human lives.

In his state-of-the-art survey Brown (2000) emphasizes that renewable natural resources are embedded in complex technological interdependencies of ecosystems and that their allocation is characterized by an "... interplay of poorly defined property rights, externalities and market failure" (p. 875). He also observes that economic models rarely extend resource interdependence beyond one or two natural resources (similar: Deacon et al. 1998) and he criticizes the propensity of economists to treat their oversimplified resource models as more than a metaphor when they offer policy advice, e.g. based on an optimal single species solution that ignores predator-prey interactions and other ecosystem interdependencies. Among Brown's (2000) prime research desiderata are increased efforts to better understand the role and function of ecosystems as well as the need to better integrate economics and ecology. Similar programmatic statements have been made by Finnoff and Tschirhart (2003a, p. 590).

While economists have a good understanding of the resource allocation mechanism in market economies by applying the economic concept of general competitive equilibrium analysis ${ }^{1}$ we are not aware of a comparable approach to the ecosystem that would be, at the same time, a suitable microfounded building block for a truly general dynamic equilibrium analysis encompassing the economy and the ecosystem as its interdependent subsystems. ${ }^{2}$ To cope with major interdependencies and feedback effects within and between the ecosystem and the economy, we suggest such an integrated general equilibrium analysis that encompasses both subsystems, the ecosystem and the economy, and treats both at the same level of analytical complexity. We address the dynamic allocation of land and nonhuman biomass with a major focus on the ecosystem model and its links to the economic

\footnotetext{
${ }^{1}$ For general equilibrium analyses applied in environmental economics we refer to Mäler (1974), Bovenberg and de Mooij (1994), Bovenberg and Goulder (1996) or Fullerton and Wolverton (2005).

${ }^{2}$ For dynamic ecological economic analyses along other lines see e.g. van den Bergh and Nijkamp (1991).
} 
submodel. Economic methodology is used to explain the interaction of species building on a small but growing literature.

Hannon (1976) formalized the notion and some implications of individual organisms optimizing net energy. Tschirhart (2000) treats such organisms as firms and is the first, to our knowledge, who models the ecosystem with optimizing individuals in a general equilibrium framework. Organisms incur energy costs, when preying biomass or sacrificing own biomass, and these transactions costs change endogenously as to equilibrate all predators' prey biomass and all preys' loss of own biomass. Finnoff and Tschirhart (2003a, 2003b) demonstate the capacity of that approach to tackle harvesting with complex intra-ecosystem interactions in numerical applied analysis. Eichner and Pethig (2005) take up Tschirhart's concept of general equilibrium in an integrated ecological-economic system (IEES). They replace the concept of equilibrating transaction costs by a complete system of competitive ecosystem markets and show that individual organisms can be interpreted as price-taking profit-maximizing firms under the roof and control of a big artificial 'agricultural firm' (biomass firm). The present paper deviates from Eichner and Pethig (2005) by recognizing in the formal model that the ecosystem is guided by an autonomous allocation mechanism of its own in which organisms are isomorphous to consumers rather than firms. Christiaans et al. (2006) model the ecosystem in isolation and determine the resource allocation in that system with the help of the competitive mechanism treating individual organisms analogous to price-taking consumers who maximize net offspring under some transactions constraints. They show that the competitive allocation mechanism, a proven powerful method of dealing with interdependencies in market economies, can be fruitfully applied to model the intertemporal allocation in the ecosystem.

In the present paper we take up the approach of Christiaans et al. (2006) to the resource allocation in the ecosystem and link the ecosystem and the economy as interdependent subsystems in an IEES where both subsystems are microfounded and treated at the same level of structural detail: individual agents optimize subject to constraints and competitive markets provide for the compatibility of individual actions (plans). ${ }^{3}$ In this setup we then focus on public consumptive ecosystem services provided by the ecosystem, biomass harvested by humans, and land converted for economic use. In our setup humans compete with all species for land and for (nonhuman) biomass, but that competition is grossly unbalanced, since humans are top predators restrained only by their perceived self-interest.

\footnotetext{
${ }^{3}$ Large parts of the ecological and bioeconomic literature focus on macro-level approaches regarding populations as the appropriate units for studying dynamic ecosystem allocations. There are also ecological models that link individual behavior to population processes (e.g. Persson and de Roos, 2003) but we are not aware of an attempt to link such models to an equilibrium model of the economy.
} 
The important feature of the IEES is that in both subsystems all (private) goods and services are traded on perfectly competitive markets. Disturbances - or shocks - in one of the subsystems cause allocative displacement effects and change relative scarcities (prices). The shocks spill over to the other subsystem causing price and quantity feedback effects although the market systems in both submodels are completely disconnected. It is a particularly important aspect of our approach, and an innovative one to our knowledge, that there is a market for land and for prey biomass in each subsystem. Since arbitrage activities between these markets are ruled out it is not clear whether and how the equilibrium prices in both market segments will differ. The answer will turn out to depend on the property rights regime of the habitat. We distinguish the scenarios of an openly accessible habitat and a privately owned habitat. In the former case biomass in situ and the habitat do not command positive economic prices while under private ownership the partition of total land into habitat and land for economic use is governed by comparing profitability.

In both scenarios efficiency requires (different) corrective taxes/subsidies to internalize the externality constituted by the consumptive ecosystem services with public good characteristics. In the case of an open access habitat additional sources of inefficiency are the divergence of prices for biomass and land in both subsystems. These extra inefficiencies can be eliminated through taxes on harvested biomass and economic land use equal to, or even exceeding the ecosystem price of the respective good.

The present approach is strong in providing rich theoretical information on (shadow) prices in the microfounded ecosystem submodel which is here tied to the general-equilibrium model of the economy and its economic prices in a rigorous and consistent way. Exploiting these features we place price tags on and determine values of all components of the ecosystem along an efficient path of the IEES with special emphasis on the role and the interplay of ecosystem prices and economic prices.

Section 2 outlines the model and section 3 characterizes the efficient allocation of that model. Section 4 introduces the concept of competitive equilibria of the IEES with open access habitat and of the IEES with privately owned habitat and presents the main results on the (in)efficiency of such general equilibria. Section 5 determines the value of the ecosystem and its components and Section 5 concludes.

\section{The model}

Consider a non-biomass natural resource whose stock $\bar{r} \in \mathbb{R}_{++}$is time-invariant and the use of which is essential for both humans and nonhuman species. We will refer to that resource 
as land because land appears to be a well fitting and very important example. ${ }^{4}$ To simplify, the use of land is assumed to be exclusive in the sense that nonhuman species (species, for short) cannot live on land used for economic purposes, and land used by species is off limits for humans. The land used by humans, $r \in[0, \bar{r}]$ is called economic land and the land used by species, $\bar{r}-r=: s$ is called habitat. The supply of economic land is expanded or reduced over time according to ${ }^{5}$

$$
\dot{r}=R\left(\begin{array}{c}
r, \mathbf{y}_{r} \\
- \\
+
\end{array}\right)
$$

where $\mathbf{y}_{r}^{\top}:=\left(y_{r 1}, \ldots, y_{r \bar{g}}\right) \in \mathbb{R}_{+}^{\bar{g}}$ denotes the vector of $\bar{g}$ (private) inputs used for maintenance and development of economic land. (1) is interpreted as the technology of a land converting firm. By definition of $s$ the habitat changes over time according to

$$
\dot{s}=-\dot{r}=-R\left(r, \mathbf{y}_{r}\right) .
$$

The habitat $s$ is home of $\bar{i}$ species whose populations are denoted by $\mathbf{n}^{\top}:=\left(n_{1}, \ldots, n_{\bar{i}}\right) \in$ $\mathbb{R}_{+}^{\bar{i}}$. Individual organisms belonging to the same species are identical. The representative individual of species $i$, called organism $i$ for short, generates net offspring

$$
b_{i}=B^{i}\left(\begin{array}{c}
s_{i}, \mathbf{z}_{i} \\
++
\end{array}\right) \quad i=1, \ldots, \bar{i}
$$

at each point in time. In (3) $s_{i} \in \mathbb{R}_{+}$denotes organism $i$ 's exclusive use of habitat resources. For example, plants occupy a patch of land that gives them (limited) access to sunlight, fresh water and nutrients. The supply of such habitat services is supposed to equal the size of the patch occupied by the organism. ${ }^{6}$

The vector $\mathbf{z}_{i}^{\top}:=\left(z_{i 1}, \ldots, z_{i i}, \ldots z_{i \bar{i}}\right) \in \mathbb{R}^{\bar{i}}$ contains all biomass transactions of organism $i$. For $i \neq j, z_{i j}$ is organism $i$ 's intake of (or in economic terms: organism $i$ 's demand for) biomass of its prey species $j$, and $z_{i i}$ is organism $i$ 's loss (or supply) of own biomass to its predators. The sign convention is $z_{i j} \geq 0$ for $i \neq j$ and $z_{i i} \leq 0$.

By definition of $b_{i}$ and $n_{i}$ the population growth turns out to be

$$
\dot{n}_{i}=n_{i} b_{i} \quad i=1, \ldots, \bar{i} .
$$

\footnotetext{
${ }^{4}$ At the expense of some stylization, other examples are water basins, water courses or air sheds.

${ }^{5}$ Upper case letters denote functions and subscripts attached to them indicate first derivatives. A plus or minus sign underneath an argument denotes the sign of the respective partial derivative.

${ }^{6}$ This setup describes land used by plants quite well but mobile animals use land in different ways. Although their land use could be modeled by introducing land services as (congestible) public goods, we refrain from that extension to keep the exposition simple.
} 
Being the top predators in the IEES, humans compete with nonhuman species for prey biomass. Let $h_{i} \geq 0$ be the biomass of species $i$ harvested by humans. $h_{i}$ is the output of the harvesting (production) function

$$
h_{i}=H^{i}\left(\begin{array}{c}
n_{i}, \mathbf{y}_{i} \\
+{ }_{+}
\end{array}\right) \quad i=1, \ldots, \bar{i}
$$

where $\mathbf{y}_{i}^{\top}:=\left(y_{i 1}, \ldots, y_{i \bar{g}}\right) \in \mathbb{R}_{+}^{\bar{g}}$ are harvesting inputs and where $H_{n_{i}}^{i}>0$ is the population stock externality known from classical harvesting models. Economic goods and services are supplied by a representative production firm using the technology

$$
F\left(\begin{array}{c}
\mathbf{h}_{f}, r_{f}, \mathbf{y}_{f} \\
+\quad+{ }_{-}
\end{array}\right) \geq 0,
$$

where $r_{f} \in \mathbb{R}_{+}$is the input of economic land services, $\mathbf{h}_{f}^{\top}:=\left(h_{f 1}, \ldots, h_{f \bar{i}}\right) \in \mathbb{R}_{+}^{\bar{i}}$ is the input of harvested biomass and where $\mathbf{y}_{f}^{\top}:=\left(y_{f 1}, \ldots, y_{f \bar{g}}\right) \in \mathbb{R}^{\bar{g}}$ is the input-output vector of goods and services. The sign convention is that $g$ is an output, if $y_{f g}>0$, and an input, if $y_{f g}<0$.

The human population of consumers is time-invariant. With all consumers being identical it suffices to consider a representative consumer whose utility is

$$
u=U\left(\begin{array}{c}
\mathbf{q}_{c}, \mathbf{y}_{c} \\
+
\end{array}\right)
$$

where $\mathbf{q}_{c}^{\top}:=\left(q_{c 1}, \ldots, q_{c \bar{k}}\right) \in \mathbb{R}^{\bar{k}}$ is a vector of public ecosystem services ${ }^{7}$ and $\mathbf{y}_{c}^{\top}:=$ $\left(y_{c 1}, \ldots, y_{c \bar{g}}\right) \in \mathbb{R}^{\bar{g}}$ is a vector of goods and services such that $y_{c g}$ is a (private) good or service for consumption, if $y_{c g}>0$, and it is a labor service supplied by the consumer, if $y_{c g}<0$. The ecosystem services are supplied by the ecosystem according to the function ${ }^{8}$

$$
\mathbf{q}=\mathbf{Q}\left(\begin{array}{c}
\mathbf{n}, r \\
+-
\end{array}\right)=\left[Q^{1}(\mathbf{n}, r), \ldots, Q^{\bar{k}}(\mathbf{n}, r)\right]
$$

\footnotetext{
${ }^{7}$ The ecosystem services considered here "... are not traded or valued in the marketplace ... [and] ... serve as public good rather than provide direct benefits to individual land owners" (Daily et al. 1997, p. 13). The consumption of these ecosystem services can alternatively be interpreted as the nonconsumptive use of renewable resources whose economic value may be substantial (Brown, 2000, p. 887).

${ }^{8} Q_{r}<0$ in (8) because according to Daily et al. (1997) relatively undisturbed land sustains the delivery of essential ecosystem services. Albers (1996) argues that preserved land can provide to neighboring economic land various benefits such as local climate effects, recreation, emission control or an enhanced view.
} 
The IEES is closed with the help of the following resource constraints:

$$
\begin{aligned}
\mathbf{y}_{f} & \geq \mathbf{y}_{c}+\sum_{i} \mathbf{y}_{i}+\mathbf{y}_{r}, \\
\mathbf{q} & \geq \mathbf{q}_{c}, \\
\mathbf{h} & \geq \mathbf{h}_{f} \\
r & \geq r_{f} \\
s & \geq \sum_{i} n_{i} s_{i}, \quad \\
\sum_{j} n_{j} z_{j i}+h_{i} & =0
\end{aligned}
$$

where $\mathbf{h}:=\left(h_{1}, \ldots, h_{\bar{i}}\right) \in \mathbb{R}_{+}^{\bar{i}}$. The resource constraint (9) is similar to that in conventional Debreu-type general equilibrium models of the economy where it is, in fact, the only resource constraint. All other constraints (10) - (14) specify and formalize the fundamental interdependence between the ecosystem and the economy: The inequality (10) forces the economy to be content with whatever ecosystem services $\mathbf{q}$ are provided by the ecosystem. The inequalities (11) and (12) constrain the economic demand for the ecosystem goods biomass and land to the amount of biomass harvested and to the land converted, respectively. The inequality (13) and the equation (14) force the nonhuman species to accommodate to the economic land use and biomass predation, respectively. (13) - (14) reflect the iron rule of the IEES, that all nonhuman species have to contend themselves with what is left after humans have set apart land and biomass for their own use.

We refrain from modeling durable human-made capital goods, capital formation and pollution since these additional features would severely raise the complexity of the analysis without providing new insights.

\section{Allocative efficiency in the IEES}

To explore the efficiency properties of a general equilibrium of the IEES, consider as a benchmark the socially optimal allocation determined by a social planner who solves the optimal control problem:

$$
\max \int_{0}^{\infty} e^{-\delta t} U\left(\mathbf{q}_{c}, \mathbf{y}_{c}\right) \mathrm{d} t \quad \text { s.t. }(1)-(14)
$$


We write down the Hamiltonian associated to that complex control problem for the purpose of keeping track of the Lagrange multipliers and co-state variables: ${ }^{9}$

$$
\begin{aligned}
\mathcal{L}^{P} & =U\left(\mathbf{q}_{c}, \mathbf{y}_{c}\right)+\sum_{i} \beta_{i} n_{i} B^{i}\left(s_{i}, \mathbf{z}_{i}\right)+(\rho-\gamma) R\left(r, \mathbf{y}_{r}\right)+\alpha_{f} F\left(\mathbf{h}_{f}, r_{f}, \mathbf{y}_{f}\right) \\
& +\sum_{k} \alpha_{q k}\left[Q^{k}(\mathbf{n}, r)-q_{k}\right]+\sum_{i} \alpha_{h i}\left[H^{i}\left(n_{i}, \mathbf{y}_{i}\right)-h_{i}\right]+\sum_{k} \alpha_{q k}^{c}\left(q_{k}-q_{c k}\right) \\
& +\sum_{g} \alpha_{y g}\left(y_{f g}-y_{r g}-\sum_{i} y_{i g}-y_{c g}\right)-\sum_{i} \alpha_{z i}\left(\sum_{j} n_{j} z_{j i}+h_{i}\right) \\
& +\sum_{i} \alpha_{v i}\left(h_{i}-h_{f i}\right)+\alpha_{r}\left(r-r_{f}\right)+\alpha_{s}\left(s-\sum_{i} n_{i} s_{i}\right) .
\end{aligned}
$$

The relevant implications of the first-order conditions of solving (15) are listed in the first column of Table $1 .{ }^{10,11}$ The existence issue is answered in ${ }^{12}$

\section{Proposition 1.}

If the function $U$ is quasi-concave and the functions $B^{i}$ (all $i$ ), $F, H^{i}$ (all $i$ ), $Q^{k}$ (all $k$ ), $R$ are concave, a solution $\left(\boldsymbol{\alpha}, \boldsymbol{\beta}, \boldsymbol{\gamma}, \boldsymbol{\rho}, \boldsymbol{A}^{m}, \boldsymbol{A}^{y}\right)$ to the Hamiltonian (15) exists.

\section{The decentralized IEES: Competitive markets in both subsystems}

As is well known, the performance of the competitive mechanism crucially depends on the specification of property rights since the emergence of competitive markets presupposes the exclusive assignment (and effective costless enforcement) of property rights. Economic goods as well as many natural resources that humans and species compete for are exclusively owned by economic agents. Yet there are also important open access resources, the commons that are the habitat of communities of species. ${ }^{13}$ We therefore proceed by distinguishing

\footnotetext{
${ }^{9}$ In view of (1) and (2) $\rho$ is the costate variable associated to $r$ and $\gamma$ is the costate variable associated to the state variable $s$.

${ }^{10}$ To simplify the exposition we assume in Table 1 that in the solution the variables $y_{r 1}, y_{i 1}, y_{f 1}, y_{c 1}$ and $z_{i 1}$ (for $i=1, \ldots, \bar{i}$ ) take on nonzero values for all $t$. The correct reading of the qualifiers "all $i$ " etc. in Table 1 is that the equation preceding such a qualifier holds for all variables $x_{i}$ whose solution values are nonzero.

${ }^{11}$ The economic interpretation of the efficiency rules listed in Table 1 is left to the reader. For some discussion in a similar context see Eichner and Pethig (2006).

${ }^{12}$ The proof of all propositions is delegated to the Appendix.

${ }^{13}$ Moreover, in many developing countries natural resources are de facto free access resources because existing property rights are poorly or not at all enforced.
} 


\begin{tabular}{|c|c|c|c|}
\hline \multicolumn{4}{|c|}{ First-order conditions for a } \\
\hline No. & social optimum & general equilibrium with & general equilibrium with \\
\hline & & open access habitat & privately owned habitat \\
\hline 1 & $\frac{R_{y_{r g}}}{R_{y_{r_{1}}}}=\frac{\alpha_{y g}}{\alpha_{y 1}} \quad$ all $g$ & $\frac{R_{y_{r g}}}{R_{y r_{1}}}=\frac{p_{y g}}{p_{y 1}} \quad$ all $g$ & $\frac{R_{y_{r g}}}{R_{y r_{1}}}=\frac{p_{y g}}{p_{y 1}} \quad$ all $g$ \\
\hline 2 & $\alpha_{v i}=\frac{\alpha_{y g}}{H_{y_{i g}}^{i}}+\alpha_{z i} \quad$ all $i$, any $g$ & $p_{h i}=\frac{p_{y g}}{H_{y_{i g}}^{i}}+\theta_{h i} \quad$ all $i$, any $g$ & $p_{h i}=\frac{p_{y g}}{H_{y_{i g}}^{i}}+\pi_{z i} \quad$ all $i$, any $g$ \\
\hline 3 & $\frac{H_{y_{i g}}^{i}}{H_{y_{i 1}}^{i}}=\frac{\alpha_{y g}}{\alpha_{y 1}} \quad$ all $i$, all $g$ & $\frac{H_{y_{i g}}^{i}}{H_{y_{i 1}}^{i}}=\frac{p_{y g}}{p_{y 1}} \quad$ all $i$, all $g$ & $\frac{H_{y_{i g}}^{i}}{H_{y_{i 1}}^{i}}=\frac{p_{y g}}{p_{y 1}} \quad$ all $i$, all $g$ \\
\hline 4 & $\frac{F_{h_{f i}}}{F_{y_{f 1}}}=\frac{\alpha_{v i}}{\alpha_{y 1}} \quad$ all $i$ & $\frac{F_{h_{f i}}}{F_{y_{f 1}}}=\frac{p_{h i}}{p_{y 1}} \quad$ all $i$ & $\frac{F_{h_{f i}}}{F_{y_{f 1}}}=\frac{p_{h i}}{p_{y 1}} \quad$ all $i$ \\
\hline 5 & $\frac{F_{r_{f}}}{F_{y_{f 1}}}=\frac{\alpha_{r}}{\alpha_{y 1}}$ & $\frac{F_{r_{f}}}{F_{y_{f 1}}}=\frac{p_{r}}{p_{y 1}}$ & $\frac{F_{r_{f}}}{F_{y_{f 1}}}=\frac{p_{r}}{p_{y 1}}$ \\
\hline 6 & $\frac{U_{y_{c g}}}{U_{y_{c 1}}}=\frac{\alpha_{y g}}{\alpha_{y 1}} \quad$ all $g$ & $\frac{U_{y_{c g}}}{U_{y_{c 1}}}=\frac{p_{y g}}{p_{y 1}} \quad$ all $g$ & $\frac{U_{y_{c g}}}{U_{y_{c 1}}}=\frac{p_{y g}}{p_{y 1}} \quad$ all $g$ \\
\hline 7 & $\frac{B_{z_{j j}}^{i}}{B_{z_{i 1}}^{i}}=\frac{\alpha_{z j}}{\alpha_{z 1}} \quad$ all $i$, all $j$ & $\frac{B_{z_{i j}}^{i}}{B_{z_{i 1}}^{i}}=\frac{\pi_{z j}}{\pi_{z 1}} \quad$ all $i$, all $j$ & $\frac{B_{z_{j j}}^{i}}{B_{z_{i 1}}^{i}}=\frac{\pi_{z j}}{\pi_{z 1}} \quad$ all $i$, all $j$ \\
\hline 8 & $\frac{B_{s_{i}}^{i}}{B_{z_{i 1}}^{i}}=\frac{\alpha_{s}}{\alpha_{z 1}} \quad$ all $i$ & $\frac{B_{s_{i}}^{i}}{B_{z_{i 1}}^{i}}=\frac{\pi_{s}}{\pi_{z 1}} \quad$ all $i$ & $\frac{B_{s_{i}}^{i}}{B_{z_{i 1}}^{i}}=\frac{\pi_{s}}{\pi_{z 1}} \quad$ all $i$ \\
\hline $\begin{array}{l}9 \mathrm{a} \\
9 \mathrm{~b}\end{array}$ & $\begin{array}{c}\dot{\rho}-\dot{\gamma}=(\rho-\gamma)\left(\delta-R_{r}\right)-\alpha_{r}-\sum_{k} U_{q_{c k}} Q_{r}^{k}+\alpha_{s} \\
\rho-\gamma=\frac{\alpha_{y g}}{R_{y_{r g}}}\end{array}$ & $\begin{array}{c}\dot{\rho}_{r}=\rho_{r}\left(\delta-R_{r}\right)-p_{r}+\theta_{r} \\
\rho_{r}=\frac{p_{y g}}{R_{y_{r g}}}\end{array}$ & $\begin{array}{c}\dot{\rho}_{r}-\dot{\gamma}_{r}=\left(\rho_{r}-\gamma_{r}\right)\left(\delta-R_{r}\right)-p_{r}+\theta_{r}+p_{s}+\theta_{s} \\
\rho_{r}-\gamma_{r}=\frac{p_{y g}}{R_{y_{r g}}}\end{array}$ \\
\hline 10 & $\begin{array}{c}\dot{\beta}_{i}=\beta_{i}\left(\delta-b_{i}\right)-\sum_{k} U_{q_{c k}} Q_{n_{i}}^{k}-\frac{\alpha_{y g} H_{n_{i}}^{i}}{H_{y_{i g}}^{i}} \\
+\alpha_{s} s_{i}+\sum_{i} \alpha_{z j} z_{i j}\end{array}$ & - & $\begin{aligned} \dot{\psi}_{i}= & \psi_{i}\left(\delta-b_{i}\right)-\theta_{n i}-\frac{p_{y g} H_{n_{i}}^{i}}{H_{y_{i g}}^{i}} \\
& +\pi_{s} s_{i}+\sum_{i} \pi_{z j} z_{i j}\end{aligned}$ \\
\hline 11 & $\frac{U_{q_{c k}}}{U_{y_{c 1}}}=\frac{\alpha_{q k}^{c}}{\alpha_{y 1}} \quad$ all $k$ & $\frac{U_{q_{c k}}}{U_{y_{c 1}}}=\frac{\lambda_{q k}}{p_{y 1}} \quad$ all $k$ & $\frac{U_{q_{c k}}}{U_{y_{c 1}}}=\frac{\lambda_{q k}}{p_{y 1}} \quad$ all $k$ \\
\hline
\end{tabular}

Table 1: Characterization of optimum and equilibrium allocations in the IEES 
two alternative scenarios of economic agents' property rights with respect to land and its uses: private ownership and open access.

Explaining the allocation in the ecosystem by means of the competitive mechanism ${ }^{14}$ also requires to implicitly defining property rights in the ecosystem: each organism will be assumed to 'own' its own biomass and a share of the habitat resource endowment (sunlight, water, nutrients). ${ }^{15}$ All organisms will then be treated analogous to economic consumers paying for purchased habitat services and prey biomass by their endowment income and their income earned by selling own biomass. Although this approach clearly is an export of conventional economic methodology, Christiaans et al. (2006) link it to the ecological literature and elaborate in some detail its plausible and - in our view - convincing ecological interpretations.

In the following subsection 4.1 the competitive mechanism for the ecosystem is formally defined and explained for predetermined human biomass harvesting and economic land use. After that the conventional competitive mechanism is introduced for the economy. Subsection 4.2 considers the case of an economy facing an open access habitat implying that biomass harvesting and land conversion is 'free of charge' (though subject to production costs). In subsection 4.3 the economy faces an ecosystem with the habitat and the economic land being privately owned such that all land uses and biomass harvesting are marketed activities.

\subsection{The competitive mechanism in the ecosystem}

In this subsection we specify the allocation mechanism in the ecosystem. Following Christiaans et al. (2006) we assume that at each point in time the allocation of land and biomasses is determined in the ecosystem by a mechanism that works as if these goods were traded on competitive markets by all organisms which are supposed to behave as if they maximize their net offspring subject to some transactions constraint. More specifically, denote by $\pi_{s} \in \mathbb{R}_{+}$the ecosystem price of land and by $\boldsymbol{\pi}_{z}^{\top}:=\left(\pi_{z 1}, \ldots, \pi_{z \bar{i}}\right) \in \mathbb{R}_{+}^{\bar{i}}$ the ecosystem prices for all species' biomass. ${ }^{16}$ For notational relief, we write $\boldsymbol{\pi}:=\left(\boldsymbol{\pi}_{z}, \pi_{s}\right)$ and we de-

\footnotetext{
${ }^{14}$ Tschirhart $(2000,2003)$ forcefully pleads for applying general equilibrium analysis to the ecosystem but he emphasizes, at the same time, that there are important differences between a general equilibrium ecosystem allocation mechanism and the (economic) competitive mechanism.

${ }^{15}$ An alternative modeling approach suggested by Pethig and Tschirhart (2001) is to assume free access to the habitat resources for all organisms.

${ }^{16}$ These prices are meant to be scarcity indicators as perceived by the organisms. To avoid clumsy wording we call them prices, nonetheless, and refer to 'ecosystem markets' although there exist neither a currency nor institutionalized markets in the ecosystem. Interestingly, these features are also missing in
} 
note by $\mathbf{K}$ the function describing an entire time path of the (possibly multidimensional) variable $\mathbf{k}$. In other words, the functional $\operatorname{sign} \mathbf{K}$ is supposed to convey the information $\mathbf{k}_{t}=\mathbf{K}(t)$ for all $t \in\left[0, \infty\left[\right.\right.$. For example, $\boldsymbol{\Pi}=\left(\boldsymbol{\Pi}^{z}, \boldsymbol{\Pi}^{s}\right)$ describes specific time paths of prices $\boldsymbol{\pi}_{t}:=\left(\boldsymbol{\pi}_{z t}, \pi_{s t}\right)$ such that $\boldsymbol{\pi}_{t}=\boldsymbol{\Pi}(t), \boldsymbol{\pi}_{z t}=\boldsymbol{\Pi}^{z}(t)$ and $\pi_{s t}=\boldsymbol{\Pi}^{s}(t){ }^{17}$ With this notation for given $\boldsymbol{\Pi}, \mathbf{H}$ and $\mathbf{R}$, and at each point in time organism $i=1, \ldots, \bar{i}$ solves

$$
\max _{\left(s_{i}, \mathbf{z}_{i}\right)} B^{i}\left(s_{i}, \mathbf{z}_{i}\right) \quad \text { s.t. } \quad \pi_{s}\left(\omega_{i}-s_{i}\right) \geq \boldsymbol{\pi}_{z}^{\top} \cdot\left(\mathbf{z}_{i}+\mathbf{h}_{i}\right)
$$

where $\mathbf{h}_{i}^{\top}:=\left(h_{i 1}, \ldots, h_{i j}, \ldots, h_{i \bar{i}}\right) \in \mathbb{R}_{+}^{\bar{i}}$ with $h_{i j} \equiv 0$ for $i \neq j$ and $h_{i i} \equiv h_{i} / n_{i}$ and where

$$
\omega_{i}=\omega_{i}(s, \mathbf{n}):=\frac{\sigma_{i} s}{\sum_{j} \sigma_{j} n_{j}} \in \mathbb{R}_{+}
$$

is organism $i$ 's endowment of or entitlement to habitat services. In $(17),\left(\sigma_{1}, \ldots, \sigma_{\bar{i}}\right)^{\top} \in \mathbb{R}_{+}^{\bar{i}}$ is a set of constant weights. By definition of $\omega_{i}(\cdot)$ it is true that $\sum_{j} n_{j} \omega_{j}(\cdot)=s$ which gives rise to the interesting interpretation that the habitat is owned by all organisms. ${ }^{18}$

To interpret (16) and (17) consider the simple case of an organism $i$ that is preyed upon and that preys itself on some species $j \neq i$ and the habitat resource, $s$. Consequently the constraint in (16) now reads

$$
\pi_{s} \omega_{i}+\pi_{z i}\left[\left(-z_{i i}\right)-\frac{h_{i}}{n_{i}}\right] \geq \pi_{s} s_{i}+\pi_{z j} z_{i j}
$$

The left side of (16') gives us the organism's total disposable income, the exogenous 'endowment income, $\pi_{s} \omega_{i}$, and the value of its sacrifice of own biomass to ecosystem predators, $-\pi_{z i} z_{i i} \geq 0$. This sacrifice is the analogue of the consumer's labor income, and the amount of own biomass harvested by humans, $h_{i} / n_{i}$, corresponds to a tax on labor income. The organism spends its total income on nutrients, $s_{i}$, and on prey biomass, $z_{i j}$, at prevailing prices. To get a better understanding of the ecological rationale of $\left(16^{\prime}\right)$ suppose first that $z_{i i}=h_{i}=0$. The organism $i$ is able to buy a bundle $\left(s_{i}, z_{i j}\right)$ worth up to $\pi_{s} \omega_{i}$ without being forced to sacrifice own biomass. ${ }^{19}$ Note that the consumption bundles $\left(s_{i}, z_{i j}\right)$ that the standard general equilibrium model of the neoclassical economy.

${ }^{17}$ Putting up with a slight misuse of notation in favor of simplicity we will denote by $\mathbf{R}, \mathbf{H}$ and $\mathbf{Q}$ the functions determining time paths of converted land, harvested biomass and ecosystem services, respectively, although these functions differ from the functions $R, H^{i}$ and $\mathbf{Q}$ in (1), (5) and (8).

${ }^{18}$ See also Christiaans et al. (2006). Note, however, that organism $i$ 's income from its entitlement to habitat services, $\pi_{s} \omega_{i}(s, \mathbf{n})$, is subject to endogenous price changes while in Christiaans et al. (2006) that income is assumed to be 'exogenous' (implying that the pertinent functions of biomass supply and demand are not homogeneous of degree zero in prices).

${ }^{19}$ Since top predators do not sacrifice own biomass they live entirely on their - supposedly large - endowment income. More generally, the higher species $i$ 's trophic level the greater tends to be its endowment $\omega_{i}$ which is therefore connotative to the notion of the species 'predation power'. 
can be purchased from the endowment income depend on the values of $\pi_{s}, n_{1}, \ldots, n_{\bar{i}}, h_{i}$ and $\pi_{z j}$ that prevail in the short-run period under consideration.

On the other hand, if we assume $\omega_{i}=h_{i}=0$ for expository purposes it is obvious that in case of positive prices $\pi_{s}$ and $\pi_{z j}$ organism $i$ cannot take up any nutrient or prey biomass unless it is prepared to sacrifice some of its own biomass. The underlying reason is that during preying or foraging organism $i$ exposes itself to its predators and the more nutrients or prey biomass organism $i$ demands the greater is the risk of being devoured and the more own biomass has to be sacrificed. The risk of being preyed upon while preying is known as the predation risk in the ecological literature (see Lima and Dik 1990) and motivates $\left(16^{\prime}\right)$.

Our preceding comments on (16') imply that when we now allow all variables in (16') to be nonzero, organism $i$ will certainly (first) take advantage of its option of riskfree foraging but it may also find it optimal to further increase its intake of nutrients and prey biomass being fully aware that more cannot be obtained without sacrificing some own biomass.

In this setup, we now define a competitive general ecological equilibrium as follows: For any given time paths of human biomass harvesting, $\mathbf{H}$, and economic land use, $\mathbf{R}$, a general ecological equilibrium is constituted by prices $\Pi$ and an ecological allocation $\mathbf{A}^{m}:=\left(\mathbf{S}^{1}, \ldots, \mathbf{S}^{\bar{i}}, \mathbf{Z}^{1}, \ldots, \mathbf{Z}^{\bar{i}}, \mathbf{Q}, \mathbf{N}\right)$ such that the solutions to all instantaneous optimization programs (16) for $\mathbf{H}$ and $\mathbf{R}$ coincide with $\mathbf{A}^{m}$ and the resource constraints (13) and (14) hold.

The general ecological equilibrium is a state where all ecosystem markets clear at each point in time and where no price-taking organism is able to increase its net offspring. To show in a more explicit way that the general ecological equilibrium consists of a sequence of short-run (or rather: instantaneous) equilibria recall that at each point in time some vector $\mathbf{v}:=(\mathbf{h}, \mathbf{n}, r)$ is given. Denote by

$$
S^{i}\left(\boldsymbol{\pi}, h_{i i}, \mathbf{n}_{i}, s\right) \quad \text { and } \quad Z^{i j}\left(\boldsymbol{\pi}, h_{i i}, \mathbf{n}_{i}, s\right)
$$

organism $i$ 's demands and supplies determined by solving (16) when prices are $\boldsymbol{\pi}:=\left(\pi_{s}, \boldsymbol{\pi}_{z}\right)$. Invoke (13) and (14) and solve the equations

$$
s=\sum_{i} n_{i} S^{i}\left(\boldsymbol{\pi}, h_{i i}, \mathbf{n}_{i}, s\right) \quad \text { and } \quad \sum_{j} n_{j} \tilde{Z}^{j i}\left(\boldsymbol{\pi}, h_{i i}, \mathbf{n}_{i}, s\right)+h_{i}=0
$$

for the ecosystem prices $\boldsymbol{\pi}^{*}$. These prices $\boldsymbol{\pi}^{*}$ clearly depend on $\mathbf{v}$ and we therefore obtain the short-run equilibrium demands and supplies as functions of $\mathbf{v}$, say $S^{i}(\mathbf{v})$ and $\mathbf{Z}^{i}(\mathbf{v})$, which determine the equilibrium net offspring $B^{i}\left[S^{i}(\mathbf{v}), \mathbf{Z}^{i}(\mathbf{v})\right]$, in turn. When the equilibrium 
net offspring is combined with (4) we obtain the system of population growth functions ${ }^{20}$

$$
\dot{n}_{i}=n_{i} B^{i}\left[S^{i}(\mathbf{v}), \mathbf{Z}^{i}(\mathbf{v})\right] \quad(\text { all } i) .
$$

We have thus demonstrated that for given $\mathbf{H}$ and $\mathbf{R}$ the time path of ecological equilibrium allocations, $\mathbf{A}^{m}$, fully determines the development of all populations over time: $\mathbf{N}=\left(\mathbf{N}^{1}, \ldots, \mathbf{N}^{\bar{i}}\right)$.

\subsection{The competitive mechanism in the IEES with an open access habitat}

First we briefly describe the competitive market economy facing an open access habitat. In that rather standard economy there are markets for all commodities, for the biomass harvested and for economic land. The corresponding market prices are $\mathbf{p}_{y}^{\top}:=\left(p_{y 1}, \ldots, p_{y \bar{g}}\right) \in$ $\mathbb{R}_{+}^{\bar{g}}, \mathbf{p}_{h}^{\top}:=\left(p_{h 1}, \ldots, p_{h \bar{i}}\right) \in \mathbb{R}_{+}^{\bar{i}}$ and $p_{r} \in \mathbb{R}_{+}$. In addition, we introduce taxes on harvested biomass, $\boldsymbol{\theta}_{h}^{\top}:=\left(\theta_{h 1}, \ldots, \theta_{h \bar{i}}\right) \in \mathbb{R}^{\bar{i}}$, on economic land, $\theta_{r} \in \mathbb{R}$, and subsidies on habitat, $\theta_{s} \in \mathbb{R}$, and on populations $\boldsymbol{\theta}_{n}^{\top}:=\left(\theta_{n 1}, \ldots, \theta_{n \bar{i}}\right) \in \mathbb{R}^{\bar{i}}$. The optimal plans of the economic agents are specified as follows:

- For given $\mathbf{P}, \mathbf{\Theta}^{r}$ and an initial stock of economic land, $r_{0}$, the land conversion firm solves:

$$
\max _{\left(\mathbf{R}, \mathbf{Y}^{r}\right)} \int_{0}^{\infty} e^{-\delta t}\left[\left(p_{r}-\theta_{r}\right) r-\mathbf{p}_{y}^{\top} \cdot \mathbf{y}_{r}\right] \mathrm{d} t \quad \text { s.t. (1) }
$$

- For given $\mathbf{P}, \Theta^{h}$ and $\mathbf{N}$ the harvesting firm solves

$$
\max _{\left(\mathbf{H}, \mathbf{Y}^{1}, \ldots, \mathbf{Y}^{\bar{i}}\right)} \int_{0}^{\infty} e^{-\delta t} \sum_{i}\left[\left(p_{h i}-\theta_{h i}\right) h_{i}-\mathbf{p}_{y}^{\top} \cdot \mathbf{y}_{i}\right] \mathrm{d} t \quad \text { s.t. (5). }
$$

- For given $\mathbf{P}$ the production firm solves

$$
\max _{\left(\mathbf{H}^{f}, \mathbf{R}^{f}, \mathbf{Y}^{f}\right)} \int_{0}^{\infty} e^{-\delta t}\left[\mathbf{p}_{y}^{\top} \cdot \mathbf{y}_{f}-\mathbf{p}_{h}^{\top} \cdot \mathbf{h}_{f}-p_{r} r_{f}\right] \mathrm{d} t \quad \text { s.t. }(6) .
$$

- For given $\mathbf{P}$ and $\mathbf{Q}$ the representative consumer solves

$$
\max _{\left(\mathbf{Y}^{c}, \mathbf{Q}^{c}\right)} \int_{0}^{\infty} e^{-\delta t} U\left(\mathbf{q}_{c}, \mathbf{y}_{c}\right) \mathrm{d} t \quad \text { s.t. } \mathbf{p}_{y}^{\top} \cdot \mathbf{y}_{c} \leq w \quad \text { and } \quad q \geq q_{c}
$$

where $w$ are lumpsum payments of profits and government transfers treated as constant by the consumers.

\footnotetext{
${ }^{20}$ Classical bioeconomic harvesting models typically assume rather than derive population growth functions of the type $\dot{n}_{i}=\hat{N}^{i}(\mathbf{n})-h_{i}$ for all $i$. The functions $N^{i}$ in (19) differ from the functions $\hat{N}^{i}$ in several important aspects: $N_{h_{i}}^{i}<0$ but $N_{h_{i}}^{i} \neq-1$ (in general) versus $\hat{N}_{h_{i}}^{i}=-1 ; N_{h_{j}}^{i} \neq 0$ (in general) versus $\hat{N}_{h_{j}}^{i}=0 ; N_{r}^{i}>0$ (in general) versus $\hat{N}_{r}^{i}=0$.
} 
In (20) - (23) all agents are modeled as non-myopic dynamic optimizers although the conversion firm is the only agent whose intertemporal plan is non-trivial. As an implication of the open-access assumption there are no markets for the biomass in situ and land available for conversion (i.e. the habitat). ${ }^{21}$ As a consequence the habitat and in situ biomass can be appropriated for free (rule of capture) by the land converting firm and the harvesting firm. Nonetheless, these firms will sell their output to the production firm at a positive price to cover their marginal costs. ${ }^{22}$

Note also that all economic agents' plans (20) - (23) are directly or indirectly linked to ecological variables: The land converting firm and the harvesting firm take land and biomass, respectively, from nonhuman species; the production firm transforms economic land and harvested biomass into consumer goods; and consumers rely on ecosystem services.

Having completed the description of the market economy we now define the competitive general equilibrium of the economy facing an open access habitat:

For any given time paths of ecosystem services, $\mathbf{Q}$, species populations, $\mathbf{N}$ and taxes $\boldsymbol{\Theta}$ a general equilibrium of the economy facing an open access habitat is constituted by prices $\mathbf{P}$ and by an economic allocation $\mathbf{A}^{y}:=\left(\mathbf{H}, \mathbf{H}^{f}, \mathbf{Q}^{c}, \mathbf{R}, \mathbf{R}^{f}, \mathbf{Y}^{c}, \mathbf{Y}^{f}, \mathbf{Y}^{r}, \mathbf{Y}^{1}, \ldots, \mathbf{Y}^{\bar{g}}\right)$ such that

- the solutions to the optimization programs (20)-(23) for $\mathbf{Q}, \mathbf{N}$ and $\mathbf{\Theta}$ coincide with $\mathbf{A}^{y}$ and

- the resource constraints (9) - (12) are satisfied.

That general equilibrium is a state where all markets are cleared at each point in time and where no price-taking agent is able to improve upon their well-being as specified by their respective objective functions. From the perspective of the IEES with open access habitat this equilibrium is partial, however, since it is conditional on predetermined $\mathbf{Q}, \mathbf{N}$ and $\boldsymbol{\Theta}$. The economic activities of land use and harvesting implied by that economic equilibrium may not be consistent with the given time paths $\mathbf{Q}, \mathbf{N}$ and $\boldsymbol{\Theta}$ on which that equilibrium has been conditioned.

So far we have introduced the concept of general equilibrium for each subsystem. Yet these subsystem equilibria are conditioned on variables determined in the other subsystem. The concept of competitive general equilibrium of the (entire) IEES with open access habitat needs to account for these interdependencies:

\footnotetext{
${ }^{21}$ The introduction of exclusive property rights for land would not matter as long as the habitat is worthless for the owner. But it will be shown in the next subsection that an owner of the habitat will be able to sell the right to harvest biomass.

${ }^{22}$ One can easily verify that if there were no costs of harvesting and land conversion and no corrective regulation, economic agents as they are modeled here would destroy the habitat and all nonhuman species.
} 
For any predetermined time path of taxes, $\boldsymbol{\Theta}$ (including the case $\boldsymbol{\Theta}(t)=0$ for all $t$ ) a general equilibrium of the IEES with open access habitat, $\left[\left(\mathbf{P}, \mathbf{A}^{y}\right),\left(\boldsymbol{\Pi}, \mathbf{A}^{m}\right)\right]$, prevails, if $\left(\mathbf{P}, \mathbf{A}^{y}\right)$ is a general equilibrium of the economy facing an open access habitat relative to $\mathbf{Q}$ and $\mathbf{N}$ and if $\left(\boldsymbol{\Pi}, \mathbf{A}^{m}\right)$ is a general ecosystem equilibrium relative to $\mathbf{H}$ and $\mathbf{R}$.

To better understand the structure of the general equilibrium of the IEES with open access habitat consider a fancy hybrid Cournot-Nash game of two Walrasian auctioneers one for each submodel. For given $\boldsymbol{\Theta}$ their strategies are $\left(\mathbf{P}, \mathbf{A}^{y}\right)$ and $\left(\boldsymbol{\Pi}, \mathbf{A}^{m}\right)$, respectively, and they attain their maximum payoff by choosing market-clearing prices in their respective submodel. Although we do not intend to formalize this 'super game' we find it useful because it highlights the existence of different and disconnected price mechanisms (reflecting decentralized decision making) in both subsystems.

The next step is to determine the first-order conditions characterizing the general equilibrium of the IEES. The Hamiltonians and Lagrangeans associated to the individual optimization programs (16) and (20) - (23) are:

$$
\begin{gathered}
\mathcal{L}^{r}=\left(p_{r}-\theta_{r}\right) r-\mathbf{p}_{y}^{\top} \cdot \mathbf{y}_{r}+\rho_{r} R\left(r, \mathbf{y}_{r}\right) \\
\mathcal{L}^{h}=\sum_{i}\left[\left(p_{h i}-\theta_{h i}\right) h_{i}-\mathbf{p}_{y}^{\top} \cdot \mathbf{y}_{i}\right]+\sum_{i} \lambda_{h i}\left[H^{i}\left(n_{i}, \mathbf{y}_{i}\right)-h_{i}\right], \\
\mathcal{L}^{y}=\mathbf{p}_{y}^{\top} \cdot \mathbf{y}_{f}-\mathbf{p}_{h}^{\top} \cdot \mathbf{h}_{f}-p_{r} r_{f}+\lambda_{f} F\left(\mathbf{h}_{f}, r_{f}, \mathbf{y}_{f}\right), \\
\mathcal{L}^{c}=U\left(\mathbf{q}_{c}, \mathbf{y}_{c}\right)+\boldsymbol{\lambda}_{q}^{\top} \cdot\left(\mathbf{q}-\mathbf{q}_{c}\right)+\lambda_{c}\left(w-\mathbf{p}_{y}^{\top} \cdot \mathbf{y}_{c}\right), \\
\mathcal{L}^{i}=B^{i}\left(s_{i}, \mathbf{z}_{i}\right)+\lambda_{i}\left[\pi_{s}\left(\omega_{i}-s_{i}\right)-\boldsymbol{\pi}_{z}^{\top} \cdot\left(\mathbf{h}_{i}+\mathbf{z}_{i}\right)\right] .
\end{gathered}
$$

The relevant implications of the first-order conditions of solving (20') - (24') are listed in the second column of Table 1. With that information we now address the question whether an efficient allocation of IEES can be sustained as a general equilibrium of the IEES with open access habitat supported by suitable taxes.

Our finding is made precise in

\section{Proposition 2.}

If the function $U$ is quasi-concave and the functions $B^{i}$ (all $i$ ), $F, H^{i}$ (all $i$ ), $Q^{k}$ (all $k$ ), $R$ are concave, there exists a distribution of profit shares and lumpsum transfers to consumers such that $\left[\left(\mathbf{P}, \mathbf{A}^{y}\right),\left(\boldsymbol{\Pi}, \mathbf{A}^{m}\right)\right]$ is an efficient general equilibrium of the IEES with open access habitat, if and only if for all $t$ prices and tax rates are assigned the values

$$
\begin{array}{ll}
\text { - } p_{h i}=\alpha_{v i}(\text { all } i), p_{r}=\alpha_{r}, p_{y g}=\alpha_{y g}(\text { all } g) & \text { (economic prices), } \\
\text { - } \pi_{z i}=\alpha_{z i}>0 \text { (all i) }, \pi_{s}=\alpha_{s} & \text { (ecosystem prices), }
\end{array}
$$




$$
\text { - } \theta_{r}=\pi_{s}-\sum_{k} \frac{p_{y g} U_{q_{c k}} Q_{r}^{k}}{U_{y_{c g}}}>0 \text { (any g), } \theta_{h i}=\pi_{z i}>0 \text { (all i) (tax rates). }
$$

With its specific assignment of prices and tax rates Proposition 2 is a decentralization result in the flavor of the second theorem of welfare economics. Yet Proposition 2 is not a fullblown generalization of that theorem from neoclassical economic models to the IEES for the following reason. The second theorem of welfare economics states, essentially, that every efficient allocation of the economy can be decentralized by prices provided the consumers' endowments and profit shares are specified and consumers receive appropriate redistributive (positive or negative) lumpsum transfers. This lumpsum redistribution is a necessary qualifier because the social planner ignores endowments and transfers in both the IEES (as evident from (15)) and the neoclassical economy. In both models lumpsum transfers to consumers are feasible. But one would also need to redistribute the organisms' endowments (17) to be able to decentralize each and every solution of (15). Yet, the redistribution of these endowments is not at the social planner's disposal because these endowments are meant to be intrinsic attributes of the organisms. This is why the second theorem of welfare economics cannot be fully extended from models of the economy to the IEES.

Proposition 2 implies that laissez-faire equilibria (defined by $\theta_{r}=\theta_{h i} \equiv 0$ ) are inefficient. There are three reasons for this inefficieny: (i) wrong economic price signals for land, (ii) wrong economic price signals for biomass and (iii) a non-internalized consumptive ecosystem services externality. ${ }^{23}$ Since efficiency requires positive tax rates the economic prices for land and biomass tend to be too low in the laissez-faire regime and therefore economic land and harvested biomass tend to be overprovided. ${ }^{24}$ In the case of biomass, the efficient tax rate is a markup on the economic biomass price equal to the ecosystem price of that biomass implying that in laissez-faire economic agents fail to account for the scarcity of biomass in the ecosystem. In the case of land conversion an analogous argument applies but now another markup on economic land is necessary to internalize the ecosystem services externality, $-\sum_{k} \frac{p_{y g} U_{q_{c k}} Q_{r}^{k}}{U_{y_{c g}}}$.

Although it is a valid conclusion from Proposition 2 that general equilibria of the IEES are inefficient, in general, if the tax rates $\Theta$ are arbitrarily chosen (e.g. with their laissez-faire values $\Theta=0$ ), it remains the question whether such equilibria exist. This question is answered in

\footnotetext{
${ }^{23}$ The model contains another two (potential) stock externalities, namely $R_{r}>0$ in (1) and $H_{n_{i}}^{i}>0$ in (5). These externalities are internalized, however, in our economy submodel owing to our simplifying assumption that there is only a single land conversion firm and a single harvesting firm.

${ }^{24}$ It is not easy to make this observation rigorous, however, because it compares two different, highly complex general equilibrium allocations.
} 


\section{Proposition 3.}

Suppose the function $U$ is quasi-concave and the functions $B^{i}$ (all $i$ ), $F, H^{i}$ (all $i$ ), $Q^{k}$ (all $k), R$ are concave. There is a set $\Omega$ of time paths of taxes $\Theta$ such that there exists a general equilibrium of the IEES with open access habitat for predetermined $\Theta$ if and only if $\Theta \in \Omega$.

\subsection{The competitive mechanism in the IEES with privately owned habitat}

In this subsection we turn to an economy with private ownership of land. We assume that the land conversion firm owns all land, the habitat as well as the economic land. It offers the economic land to the production firm at price $p_{r} \in \mathbb{R}_{+}$and it offers the right to harvest biomass in the habitat at price $p_{s} \in \mathbb{R}_{+}$per unit of land to the harvesting firm. The conversion firm's strategy to convert habitat into economic land or vice versa depends on the relative profitability of both kinds of land uses. Having full information about the ecosystem dynamics that we described in subsection 4.1 the harvesting firm is assumed to take into account the impact of her harvesting activities on population growth.

While the optimal plans of the consumer and the producer remain as in (22) and (23), the optimal plans of the land conversion firm and the harvesting firm are now given by:

- For given $\mathbf{P}, \boldsymbol{\Theta}^{r}, \boldsymbol{\Theta}^{s}$ and an initial stock of economic land, $r_{0}$, the land conversion firm solves:

$$
\max _{\left(\mathbf{R}, \mathbf{S}, \mathbf{Y}^{r}\right)} \int_{0}^{\infty} e^{-\delta t}\left[\left(p_{r}-\theta_{r}\right) r+\left(p_{s}+\theta_{s}\right) s-\mathbf{p}_{y}^{\top} \cdot \mathbf{y}_{r}\right] \mathrm{d} t \quad \text { s.t. (1), (2). }
$$

- For given $\mathbf{P}, \mathbf{\Theta}^{n}$ and initial stocks of populations $n_{i 0}$ the harvesting firm solves

$$
\begin{aligned}
\max _{\left(\mathbf{H}, \mathbf{Y}^{1}, \ldots, \mathbf{Y}^{\bar{i}}, \mathbf{N}, \mathbf{S}^{1}, \ldots, \mathbf{S}^{\bar{i}}, \mathbf{Z}^{1}, \ldots, \mathbf{Z}^{\bar{i}}, \boldsymbol{\Pi}\right)} & \int_{0}^{\infty} e^{-\delta t}\left[\sum_{i}\left(p_{h i} h_{i}+\theta_{n i} n_{i}\right)-p_{s} s-\mathbf{p}_{y}^{\top} \cdot \mathbf{y}_{i}\right] \mathrm{d} t \\
& \text { s.t. (3), (4), (5), (18). }
\end{aligned}
$$

A closer look at (25) shows that the organisms' demand and supply functions (18), the net offspring function (4) and the population dynamics (5) are constraints and that the ecosystem prices are decision variables in the harvesting firm's program of maximizing of present value profits. The harvesting firm respects and accounts for the optimizing behavior of all organisms as well as the ecosystem law of eating and being eaten. Unlike ranchers who feed their lifestock and shelter it against predators the harvesting firm in the present model chooses its optimal intertemporal harvesting strategy by taking all 'natural' ecosystem interactions into account. It thus differs greatly from the social planner who 
calculates the solution to (15) and then assigns a consumption, production and feeding program, respectively, to the consumers, producers and organisms without any regard to 'autonomous' optimizing behavior of individual agents. The harvesting firm is modelled here as a Stackelberg leader with the organisms being Stackelberg followers. It accounts for the organism's "reaction functions" (here demand and supply functions (18)) inducing the organisms to choose the ecological allocation implied by (16). ${ }^{25}$

Having specified the economic agents optimization programs we are now in the position to define the competitive general equilibrium of the IEES with privately owned habitat: ${ }^{26}$ For any given time path of ecosystem services $\boldsymbol{Q}$ and taxes $\boldsymbol{\Theta}$ (including the case $\boldsymbol{\Theta}(t)=0$ for all $t$ ) a general equilibrium of the private ownership IEES is constituted by prices $(\mathbf{P}, \boldsymbol{\Pi})$ and an allocation $\left(\mathbf{A}^{m}, \mathbf{A}^{y}\right)$ such that

- the solutions to the optimization programs (22) - (25) for $\mathbf{Q}$ and $\mathbf{\Theta}$, coincide with $\left(\mathbf{A}^{y}, \mathbf{A}^{m}\right)$ and

- the resource constraints (9) - (14) are satisfied.

Following the procedure of the last subsection we solve the Hamiltoneans and Lagrangeans $\left(22^{\prime}\right),\left(23^{\prime}\right)$ and

$$
\begin{gathered}
\mathcal{L}^{r}=\left(p_{r}-\theta_{r}\right) r+\left(p_{s}+\theta_{s}\right) s-\mathbf{p}_{y}^{\top} \cdot \mathbf{y}_{r}+\left(\rho_{r}-\gamma_{r}\right) R\left(r, \mathbf{y}_{r}\right), \\
\mathcal{L}^{h}=\sum_{i} p_{h i} h_{i}-p_{s} s-\mathbf{p}_{y}^{\top} \cdot \mathbf{y}_{i}+\sum_{i} \theta_{n i} n_{i}+\sum_{i} \lambda_{h i}\left[H^{i}\left(n_{i}, \mathbf{y}_{i}\right)-h_{i}\right] \\
+\sum_{i} \psi_{i} n_{i} B^{i}\left(s_{i}, z_{i}\right)+\sum_{i} \lambda_{s i} n_{i}\left[S^{i}\left(\boldsymbol{\pi}, h_{i i}, \mathbf{n}, s\right)-s_{i}\right] \\
+\sum_{j} \sum_{i} \lambda_{z i j} n_{i}\left[Z^{i j}\left(\boldsymbol{\pi}, h_{i i}, \mathbf{n}, s\right)-z_{i j}\right]
\end{gathered}
$$

and obtain the first-order conditions listed in the third column of Table $1{ }^{27}$ The efficiency implications are summarized in

\section{Proposition 4.}

If the function $U$ is quasi-concave and the functions $B^{i}$ (all $\left.i\right), F, H^{i}$ (all $i$ ), $Q^{k}$ (all $k$ ), $R$

\footnotetext{
${ }^{25}$ Obviously, the informational requirements on the part of the harvesting firm of solving (25) are extremely demanding which characterizes that procedure as a polar case. An opposite polar case has been modeled in the previous subsection since under open access the ecosystem dynamics have been no part of any economic agent's optimization calculus. In the scenario of private habitat ownership incomplete information on the complex ecosystem dynamics would appear to be realistic. Yet an analysis of decision making under uncertainty is beyond the scope of the present paper.

${ }^{26}$ When the habitat is privately owned the equilibrium of the IEES cannot be decomposed anymore into equilibria of its subsystems.

${ }^{27}$ The derivation of rows $2,3,7,8$ and 10 in column 3 can be found in the Appendix.
} 
are concave, there exists a distribution of profit shares and lumpsum transfers to consumers such that $\left[\left(\mathbf{P}, \mathbf{A}^{y}\right),\left(\mathbf{\Pi}, \mathbf{A}^{m}\right)\right]$ is an efficient general equilibrium of IEES with privately owned habitat, if and only if for all $t$ prices and tax rates are assigned the values

- $p_{h i}=\alpha_{v i}($ all $i), p_{r}=\alpha_{r}, p_{s}=\alpha_{s}, p_{y g}=\alpha_{y g}($ all g)

(economic prices),

- $\pi_{z i}=\alpha_{z i}>0$ (all i), $\pi_{s}=\alpha_{s}$ (ecosystem prices),

- $\theta_{r}\left[\right.$ or $\left.\theta_{s}\right]=-\sum_{k} \frac{p_{y g} U_{q_{c k}} Q_{r}^{k}}{U_{y c g}}>0$ (any g), $\theta_{n i}=\sum_{k} \frac{p_{y g} U_{q_{c k}} Q_{n_{i}}^{k}}{U_{y_{c g}}}>0$ (all i, any g) (tax rates, subsidies).

As in the IEES with open access habitat the laissez-faire equilibria $\left(\theta_{r}=\theta_{s}=\theta_{n i}=0\right)$ of the IEES with privately owned habitat are inefficient, too. While the price signals for biomass are now right there remain two sources for inefficiency: (i) a non-internalized ecosystem service externality of habitat, (ii) non-internalized ecosystem service externalities of populations. In the laissez-faire equilibrium allocation the habitat and the populations tend to be too small. These inefficiencies can be corrected by subsidizing the habitat and the populations. Obviously, instead of inducing the land conversion firm to extend the habitat through a subsidy the government could also tax economic land thus providing an incentive for the land conversion firm to convert less land. Comparing Propositions 2 and 4 elucidates that under both scenarios the government could levy a tax on economic land but under open access the land tax corrects for ecosystem service externalities and for the ecosystem price of land, $\pi_{s}$, whereas the latter emerges in the private ownership economy in form of the habitat price $p_{s}=\pi_{s}$ paid by the harvesting firm. Finally, it is interesting to observe that in the economy facing a privately owned habitat population subsidies are applied whereas in the economy facing an open access habitat harvesting taxes are needed to implement the efficient allocation. Hence under different property rights scenarios different corrective policy instruments are called for. Finally, the existence issue is addressed in

\section{Proposition 5.}

Suppose the function $U$ is quasi-concave and the functions $B^{i}$ (all $i$ ), F, $H^{i}$ (all $i$ ), $Q^{k}$ (all $k$ ), $R$ are concave. There is a set $\Upsilon$ of time paths of taxes $\Theta$ such that there exists a general equilibrium of the IEES with privately owned habitat for predetermined $\Theta$ if and only if $\Theta \in \Upsilon$.

\section{The value of the ecosystem and its components}

In the remainder of the paper we will determine resource prices and/or values along the efficient path that has been characterized in Table 1 combined with the Propositions 2 
and 4, respectively. We will use the pertaining marginal efficiency conditions for valuing the components of the ecosystem with a special emphasis on the role and interplay of ecosystem prices and economic prices. Exploiting that information in a systematic way will prove the present approach to be a powerful basis for putting price tags on ecosystem components. The reason is not only because the information our microfounded generalequilibrium approach to the ecosystem provides on scarcities or (shadow) prices in the ecosystem is much richer than that in conventional macro-level models of population ecology but also because the submodel ecosystem is here tied to a general-equilibrium economy and its economic prices in a rigorous and consistent way. Observe also that the following valuation exercises do not depend on the property rights regime of the habitat since the focus is on efficient allocations. Either scenario is covered when the appropriate efficiencyrestoring taxes are accounted for that have been specified in the Propositions 2 and 4, respectively.

\section{Proposition 6.}

(i) In an efficient general equilibrium of the IEES

- the economic price of biomass of species $i$ is

$$
p_{h i}=\pi_{z i}+\underbrace{\frac{p_{y g}}{H_{y_{i g}}^{i}}}_{[1]} \quad \text { all } i, \text { any } g \text {, }
$$

- the economic price of land services is

$$
p_{r}=\pi_{s}-\underbrace{\sum_{k} \frac{p_{y g} U_{q_{c k}} Q_{r}^{k}}{U_{y_{c g}}}}_{[2]}+\underbrace{\frac{p_{y g}}{R_{y_{r g}}}\left[\delta-\left(\frac{p_{y g}}{R_{y_{r g}}}\right) \frac{R_{y_{r g}}}{p_{y g}}\right]}_{[3]}-\underbrace{\frac{p_{y g} R_{r}}{R_{y_{r g}}}}_{[4]} \text { any } g .
$$

(ii) In a steady state of the efficient general equilibrium of the IEES

- the price of (a living organism of) species $i$ is

$$
\beta_{i}^{*}=\frac{1}{\delta}(\underbrace{\sum_{k} \frac{p_{y g}^{*} U_{q_{c k}} Q_{n_{i}}^{k}}{U_{y_{c g}}}}_{[5]}-\underbrace{\pi_{s}^{*} \omega_{i}}_{[6]}+\underbrace{\frac{\varepsilon\left(h_{i}, n_{i}\right)\left(p_{h i}^{*}-\pi_{z i}^{*}\right) h_{i}}{n_{i}}}_{[7]}+\underbrace{\frac{\pi_{z i}^{*} h_{i}}{n_{i}}}_{[8]})
$$

where $\varepsilon\left(h_{i}, n_{i}\right):=n_{i} H_{n_{i}}^{i} / h_{i}>0$; 
- the price of (a unit of) economic land is

$$
\rho^{*}=\frac{1}{\delta-R_{r}}(p_{r}^{*}-\frac{\pi_{s}^{*} R_{r}}{\delta}+\underbrace{\sum_{k} \frac{p_{y g}^{*} U_{q_{c k}} Q_{r}^{k}}{U_{y_{c g}}}}_{[2]}) \quad \text { any } g,
$$

- the price of (a unit of) habitat is

$$
\gamma^{*}=\frac{\pi_{s}}{\delta}
$$

To capture the essence of (26) recall that 'harvesting' of prey biomass by predators in the ecosystem is costless whereas human predators incur positive marginal harvesting costs [1]. With zero marginal costs of harvesting, i.e. with $H_{y_{i g}}^{i} \rightarrow \infty$, (26) yields $p_{h i}=\pi_{z i}$. Hence efficiency requires the biomass price of all species $i$ to be the same in both submodels for all species $i$ that are harvested unless asymmetric conditions in both markets warrant a price difference. Although (27) looks much more complex than (26), the same argument applies for the prices of land services in both subsystems. In [4] the stock externality $R_{r}<0$ increases the marginal conversion costs, $p_{y g} / R_{y_{r g}}$, that are positive in the economy but have no equivalent in the ecosystem. To rule out marginal conversion costs as a cause of divergence suppose that $R_{y_{r g}} \rightarrow \infty$. This assumption eliminates [4] as well as the dynamic marginal costs [3]. The remaining factor [2] that renders different the efficient land prices in both submodels represents the external marginal costs generated by the ecosystem services externality which exists in the economy but has no counterpart in the ecosystem. In fact, if that externality is 'switched off' (for the sake of the argument) by setting $U_{q_{c k}} \equiv 0$, then (27) is turned into $p_{r}=\pi_{s}$. In other words, efficiency requires land services to be uniformly priced in both subsystems unless these subsystems exhibit differences in internal and external marginal costs.

Dynamic marginal land conversion costs [3] accrue in the economy but not in the ecosystem because humans determine the size of habitat unilaterally and the land conversion firm controls for the time path of economic land use. The dynamic marginal costs comprise two components. $\frac{\delta p_{y g}}{R_{y g}}$ reflects that increasing land conversion diminishes the available land stock. $-\left(\frac{\dot{p_{y g}}}{R_{y_{g}}}\right)$ captures the costs of keeping land in stock. If $\left(\frac{\dot{p_{y g}}}{R_{y_{g}}}\right)>0$, the market value of economic land increases and the land converting firm will keep the land in stock. If $\left(\frac{p_{y g}}{R_{y_{g}}}\right)<0$ the market value of the stock decreases and it may be expensive not to convert land in good time.

$\beta_{i}^{*}$ from (28) is the price of a living organism $i$ in the long-run equilibrium that is made up of the present value of the components [5] - [8]. The term [5] represents the external 
benefits of the marginal organism $i$ in form of enhancing the provision of public ecosystem services; [7] is the marginal benefit of the stock externality $H_{n_{i}}^{i}>0$; [8] gives us the value of organism $i$ 's biomass loss due to harvesting. The value $\beta_{i}^{*}$ is reduced by the value of organism $i$ 's land endowment [6] which represents the opportunity costs of economic land use.

In $(29), \rho^{*}$ is the efficient steady state price of economic land. It equals the present value of the price of economic land services, $p_{r}^{*} /\left(\delta-R_{r}\right)$, (where the relevant discount rate is not $\delta$ but $\left(\delta-R_{r}\right)>\delta$ ) reduced by two corrective factors: the present value of the opportunity costs of the stock externality, $\pi_{s}^{*} R_{r} / \delta\left(\delta-R_{r}\right)$, and the present value of the term [2] that has already been described above. Finally, the price $\gamma^{*}$ of the habitat is simply the present value of the ecosystem price for land.

Based on Proposition 6 (ii) we are able to calculate the value of the ecosystem in an efficient steady state. The total value of all species is

$$
\sum_{i} \beta_{i}^{*} n_{i}=\frac{1}{\delta}[\underbrace{\sum_{i} \sum_{k} \frac{p_{y g}^{*} U_{q_{c k}} Q_{n_{i}}^{k}}{U_{y_{c g}} n_{i}}}_{[9]}-\underbrace{\pi_{s}^{*} s}_{[10]}+\underbrace{\sum_{i} \varepsilon\left(h_{i}, n_{i}\right)\left(p_{h i}^{*}-\pi_{z i}^{*}\right) h_{i}}_{[11]}+\underbrace{\sum_{i} \pi_{z i}^{*} h_{i}}_{[12]}] .
$$

That value is composed of

- the species' contribution to the aggregate marginal benefits of public ecosystem services [9] minus

- the total value (at the ecosystem price of land services) of the habitat [10] plus

- the aggregate value of all stock externalities in harvesting [11] plus

- the total value (at ecosystem prices) of all biomass harvested by humans [12].

From (29) - (31) it is straightforward to compute the total value of the ecosystem in an efficient steady state as $^{28}$

$$
\sum_{i} \beta_{i}^{*} n_{i}+\gamma^{*} s=\frac{1}{\delta}\left[\sum_{i} \sum_{k} \frac{p_{y g}^{*} U_{q_{c k}} Q_{n_{i}}^{k}}{U_{y_{c g}} n_{i}}+\sum_{i} \varepsilon\left(h_{i}, n_{i}\right)\left(p_{h i}^{*}-\pi_{z i}^{*}\right) h_{i}+\sum_{i} \pi_{z i}^{*} h_{i}\right]
$$

and the value of the entire stock of land as

$$
\rho^{*} r+\gamma^{*} s=\frac{1}{\delta-R_{r}}(\underbrace{p_{r}^{*} r}_{[13]}+\pi_{s}^{*} s\left(1-\frac{(s-r) R_{r}}{\delta s}\right)+\underbrace{\sum_{k} \frac{p_{y g}^{*} U_{q_{c k}} Q_{r}^{k}}{U_{y_{c g}}} r}_{[14]}) .
$$

\footnotetext{
${ }^{28}$ Our expression (32) provides a theoretical underpinning of Constanza et al. (1997)'s empirical valuation of the world's ecosystem services.
} 
It is interesting to observe that the value of habitat [10] cancels out when deriving the value of the ecosystem (32). Suppressing stock externalities, formally $H_{n_{i}}=R_{r} \equiv 0$, the formulas (32) and (33) have straightforward and appealing interpretations. The value of the ecosystem then consists of the benefits from ecosystem services [9] and from the value of biomass harvested [12]. The value of the entire stock of land is then composed of the value of economic land services [13] plus the value of habitat [10] minus the external cost of economic land use [14].

\section{Concluding remarks}

The main purpose of the present paper is methodological and conceptual but it aims, at the same time, to demonstrate that the application of a general-equilibrium competitive allocation mechanism to a microfounded integrated ecological-economic system yields specific and new insight with regard to efficient pricing of the ecosystem and its services. The ecosystem and the economy are designed as interdependent submodels and the focus is on intra-system interactions of individuals as well as on inter-system repercussions of these interactions. Both submodels are characterized by resource scarcity and a decentralized and uncoordinated mode of allocating resources. The competitive mechanism therefore appears to be an appropriate methodological device to 'coordinate' the activities of optimizing agents through prices. For economists, the ultimate way of assessing scarcity and value is to put a price tag on the items under consideration. On the conceptual level we have shown that our approach generates such prices in both submodels, and that it is able to determine and compare the prices of goods, namely land services and biomass, that are traded in both subsystems on competitive but disconnected markets.

As in other studies, in our analysis the source of inefficiencies remains, of course, the "... interplay of properly defined property rights, externalities and market failure" (Brown 2000, p. 875). The existence of exclusive property rights for the habitat or their absence also plays a similar role in that context as in conventional studies. Yet our approach allows a fresh diagnosis by linking ecosystem prices and economic prices as well as ecosystem prices and the rates of corrective taxes. In the laissez-faire equilibrium of the IEES with open access habitat economic agents disregard the ecosystem prices of land and biomass (as they disregard other non-market spillovers) which calls for corrective regulation. We have shown that there are taxes on economic land use and taxes on harvested biomass that are capable to restore efficiency. Quantity (rather than price) regulation clearly is an alternative way toward allocative efficiency being applied in practice in form of harvesting quotas, land use restrictions, land zoning etc. 
Although the paper does not focus on applied techniques of valuating ecosystem components, our approach has the potential to serve as a basis for a new kind of applied general equilibrium analyses. By now, such analyses have become a standard tool for simulating the incidence of economic policies with significant ramifications in many economic markets. With appropriate parametrizations and calibrations of both the economic and the ecosystem submodel ${ }^{29}$ one would be able to run numerical simulations in a large-scale IEES to gauge the quantitative impact on the whole system of alternative policies of biomass harvesting and economic land use. Such applications would generate numerical information on ecosystem prices and values that would be an important input in designing efficient natural resource policies.

\section{References}

Albers H.J. (1996), Modeling ecological constraints on tropical forest management: spatial interdependence, irreversibility, and uncertainty, Journal of Environmental Economics and Management 30, 73-94.

Alcamo J. et al. (2003), Millenium Ecosystem Assessment - Ecosystems and Human Well-Being: A Framework for Assessment, Island Press, Washington.

Bovenberg A.L. and R. de Mooij (1994), Environmental levies and distortionary taxation, American Economic Review 84, 1085-1089.

Bovenberg A.L. and H.L. Goulder (1996), Optimal environmental taxation in the presence of other taxes: general equilibrium analyses, American Economic Review 86, 985-1000.

Brown G.M. (2000), Renewable natural resource management and use without markets, Journal of Economic Literature 30, 73-94.

Christiaans T., Eichner T. and R. Pethig (2006), A micro-level 'consumer approach' to species population dynamics, Natural Resource Modeling, in press. http://www.ces ifogroup.de/pls/guestci/download/CESifo\%20Papers\%20August\%202005/cesifo 1_wp1530.pdf

Constanza et al. (1997), The value of the world's ecosystem services, Nature 387, 253-260.

Daily G.C. et al. (1997), Ecosystem services: benefits supplied to human societies by natural ecosystems, Issues in Ecology 2, (Official publication of the Ecological Society

\footnotetext{
${ }^{29}$ In different but related frameworks Finnoff and Tschirhart (2003a, 2003b) calibrate a fairly complex general equilibrium model of the ecosystem and study the repercussions of harvesting strategies in numerical analysis.
} 
of America).

Deacon et al. (1998), Research trends and opportunities in environmental and natural resource economics, Environmental and Resource Economics 11, 383-397.

Eichner T. and R. Pethig (2005), Ecosystem and economy: An integrated dynamic general equilibrium approach, Journal of Economics 85, 213-249.

Eichner T. and R. Pethig (2006), Nonanthropocentric nature protection, Social Choice and Welfare 26, 47-74.

Finnoff D. and J. Tschirhart (2003a), Harvesting in an eight-species ecosystem, Journal of Environmental Economics and Management 45, 589-611.

Finnoff D. and J. Tschirhart (2003b), Protecting an endangered species while harvesting its prey in a general equilibrium ecosystem model, Land Economics 79, 160-180.

Fullerton D. and A. Wolverton (2005), The two-part instrument in a second best world, Journal of Public Economics 89, 1961-1975.

Hannon B. (1976), Marginal product pricing in the ecosystem, Journal of Theoretical Biology 56, 253-267.

Lima S.L. and L.M. Dik (1990), Behavioral decisions made under the risk of predation: A review and prospectus, Canadian Journal of Zoology 68, 619-640.

Mäler K.-G. (1974), Environmental economics: A theoretical inquiry, John Hopkins University Press for Resources for the future, Baltimore.

Persson L. and A.M. De Roos (2003), Adaptive habitat use in size-structured populations: Linking individual behavior to population processes, Ecology 84, 1129-1139.

Pethig R. and J. Tschirhart (2001), Microfoundations of population dynamics, Journal of Bioeconomics 3, 27-49.

Seierstad A. and K. Sydsæter (1987), Optimal control theory with economic applications, North-Holland, Amsterdam et al.

Tschirhart J. (2000), General equilibrium of an ecosystem, Journal of Theoretical Biology 203, 13-32.

Tschirhart J. (2003), Ecological transfers in non-human communities parallel economic markets in a general equilibrium ecosystem model, Journal of Bioeconomics 5, 193214. 
van den Bergh J.C.J.M and P. Nijkamp (1991), Operationalizing sustainable development: dynamic ecological economic models, Ecological Economics 4, 11-33.

\section{Appendix}

\section{Proof of Proposition 1:}

Proposition 1 follows from applying Theorem 11 in Seierstad and Sydsæter (1987, chapter 6, p. 385). We refrain from tracing the requirements of that Theorem 11 in all detail. However, the main substantive conditions are listed in Proposition 1.

\section{Derivation of rows $2,3,7,8$ and 10 in column 3 of Table 1:}

The first-order conditions for the harvesting firm's problem (21") are

$$
\begin{aligned}
\mathcal{L}_{h_{i}}^{h} & =p_{h i}-\lambda_{h i}+\lambda_{s i} S_{h_{i i}}^{i}+\sum_{j} \lambda_{z i j} Z_{h_{i i}}^{i j}=0, \\
\mathcal{L}_{s}^{h} & =-p_{s}+\sum_{i} \lambda_{s i} n_{i} S_{s}^{i}+\sum_{j} \sum_{i} \lambda_{z i j} n_{i} Z_{s}^{i j}=0, \\
\mathcal{L}_{y_{i g}}^{h} & =-p_{y g}+\lambda_{h i} H_{y_{i g}}^{i}=0, \\
\mathcal{L}_{z_{i j}}^{h} & =\psi_{i} n_{i} B_{z_{i j}}^{i}-\lambda_{z i j} n_{i}=0, \\
\mathcal{L}_{s_{i}}^{h} & =\psi_{i} n_{i} B_{s_{i}}^{i}-\lambda_{s i} n_{i}=0, \\
\mathcal{L}_{\pi_{z i}}^{h} & =\sum_{j} \lambda_{s j} n_{j} S_{\pi_{z i}}^{j}+\sum_{k} \sum_{j} \lambda_{z j k} n_{j} Z_{\pi_{z i}}^{j k}=0, \\
\mathcal{L}_{\pi_{s}}^{h} & =\sum_{j} \lambda_{s j} n_{j} S_{\pi_{s}}^{j}+\sum_{k} \sum_{j} \lambda_{z j k} n_{j} Z_{\pi_{s}}^{j k}=0, \\
\dot{\psi}_{i} & =\left(\delta-b_{i}\right) \psi_{i}-\lambda_{h i} H_{n_{i}}^{i}-\theta_{n i}-\sum_{j} \lambda_{s j} n_{j} S_{n_{i}}^{j}-\sum_{j} \sum_{k} \lambda_{z j k} n_{j} Z_{n_{i}}^{j k} .
\end{aligned}
$$

Equations (34c)-(34f) can be rearranged to

$$
\begin{aligned}
\frac{H_{y_{i g}}^{i}}{H_{y_{i 1}}^{i}} & =\frac{p_{y g}}{p_{y 1}}, \\
\frac{B_{z_{i j}}^{i}}{B_{z_{i 1}}^{i}} & =\frac{\lambda_{z i j}}{\lambda_{z i 1}}, \\
\frac{B_{s_{i}}^{i}}{B_{z_{i 1}}^{i}} & =\frac{\lambda_{s i}}{\lambda_{z i 1}} .
\end{aligned}
$$

Solving the organism $i$ 's optimization problem (16) we obtain

$$
\begin{aligned}
\frac{B_{z_{i j}}^{i}}{B_{z_{i 1}}^{i}} & =\frac{\pi_{z j}}{\pi_{z 1}}, \\
\frac{B_{s_{i}}^{i}}{B_{z_{i 1}}^{i}} & =\frac{\pi_{s}}{\pi_{z 1}} .
\end{aligned}
$$


From (36)-(37) and (38)-(39) we infer

$$
\frac{\lambda_{z i j}}{\lambda_{z i 1}}=\frac{\pi_{z j}}{\pi_{z 1}} \quad \text { and } \quad \frac{\lambda_{s i}}{\lambda_{z i 1}}=\frac{\pi_{s}}{\pi_{z 1}} \quad \forall i .
$$

Taking into account that both at the social optimum and in the market we can choose biomass of species 1 as numeraire $\left(\lambda_{z i 1}=\pi_{z 1} \equiv 1\right)$ establishes $\lambda_{z i j}=\pi_{z j}$ and $\lambda_{s i}=\pi_{s}$ for all $i$. Next, we use the species budget constraint $\pi_{s}\left(\omega_{i}-s_{i}\right)=\sum_{j} \pi_{z j} z_{i j}+\pi_{z i} h_{i i}$, the demand and supply functions $S^{i}\left(\boldsymbol{\pi}, h_{i i}, \mathbf{n}_{i}, s\right), Z^{i j}\left(\boldsymbol{\pi}, h_{i i}, \mathbf{n}_{i}, s\right)$ and the definition of the endowments $\omega_{i}:=\frac{\sigma_{i} s}{{ }_{j} \sigma_{j} n_{j}}$ to obtain

$$
\frac{\pi_{s} \sigma_{i} s}{\sum_{j} \sigma_{j} n_{j}}-\pi_{s} S^{i}\left(\boldsymbol{\pi}, h_{i i}, \mathbf{n}_{i}, s\right)-\sum_{j} \pi_{z j} Z^{i j}\left(\boldsymbol{\pi}, h_{i i}, \mathbf{n}_{i}, s\right)-\pi_{z i} h_{i i}=0
$$

Multiply equation (41) by $n_{i}$ and sum over $i$ yields

$$
A:=\pi_{s} s-\sum_{i} \pi_{s} n_{i} S^{i}\left(\boldsymbol{\pi}, h_{i i}, \mathbf{n}_{i}, s\right)-\sum_{j} \sum_{i} n_{i} \pi_{z j} Z^{i j}\left(\boldsymbol{\pi}, h_{i i}, \mathbf{n}_{i}, s\right)-\sum_{i} \pi_{z i} h_{i}=0
$$

Next, differentiation of (42) with respect to $h_{i}, s, \pi_{z i}, \pi_{s}$, and $n_{i}$ one gets

$$
\begin{aligned}
\frac{\mathrm{d} A}{\mathrm{~d} h_{i}} & =-\pi_{s} S_{h_{i i}}^{i}-\sum_{j} \pi_{z i} Z_{h_{i} i}^{i j}-\pi_{z i}=0, \\
\frac{\mathrm{d} A}{\mathrm{~d} s} & =\pi_{s}-\sum_{i} \pi_{s} n_{i} S_{s}^{i}-\sum_{j} \sum_{i} \pi_{z j} n_{i} Z_{s}^{i j}=0, \\
\frac{\mathrm{d} A}{\mathrm{~d} \pi_{z i}} & =-\sum_{i} \pi_{s} n_{i} S_{\pi_{z i}}^{i}-\sum_{j} \sum_{i} \pi_{z j} n_{i} Z_{\pi_{z i}}^{i j}-h_{i}-\sum_{j} n_{j} z_{j i}=0, \\
\frac{\mathrm{d} A}{\mathrm{~d} \pi_{s}} & =s-\sum_{i} n_{i} s_{i}-\sum_{i} \pi_{s} n_{i} S_{\pi_{s}}^{i}-\sum_{j} \sum_{i} \pi_{z j} n_{i} Z_{\pi_{s}}^{i j}=0, \\
\frac{\mathrm{d} A}{\mathrm{~d} n_{i}} & =-\pi_{s} s_{i}-\sum_{j} \pi_{s} n_{j} S_{n_{i}}^{j}-\sum_{j} \pi_{z j} z_{i j}-\sum_{j} \sum_{k} \pi_{z k} n_{j} Z_{n_{i}}^{i k}=0 .
\end{aligned}
$$

Finally, combining (34a) and (43a), (34b) and (43b), (34f) and (43c), (34g) and (43d), (34h) and (43e), results in

$$
\begin{aligned}
p_{h i} & =\frac{p_{y g}}{H_{y_{i g}}^{i}}+\pi_{z i}, \\
p_{s} & =\pi_{s} \\
h_{i}+\sum_{j} n_{j} z_{j i} & =0 \\
s & =\sum_{i} n_{i} s_{i}, \\
\dot{\psi}_{i} & =\left(\delta-b_{i}\right) \psi_{i}-\frac{p_{y g} H_{n_{i}}^{i}}{H_{y_{i g}}^{i}}-\theta_{n i}+\pi_{s} s+\sum_{j} \pi_{z j} z_{i j} .
\end{aligned}
$$




\section{Proof of Proposition 2:}

Observe that the quasi-concavity of $U$ and the concavity of the functions $B^{i}$ (all $i$ ), $F, H^{i}$ (all $i), Q^{k}$ (all $k$ ), $R$ ensures that the first-order conditions listed in the colum 1 (2) of Table 1 are both necessary and sufficient for the efficient (open acces IEES) allocation. To prove Proposition 2 check that with the assignment of prices and taxes as listed in Proposition 2 there is an exact correspondence between the second column of Table 1 and the first column of Table 1.

\section{Proof of Proposition 3:}

If for some predetermined time path of taxes, $\Theta$, a general equilibrium of the IEES with open access habitat exists, the associated equilibrium allocation entails specific values of the externality-related variables $\mathbf{H}$ and $\mathbf{R}$, say $\overline{\mathbf{H}}$ and $\overline{\mathbf{R}}$. Rather than proving existence for predetermined $\Theta$ we turn the problem on its head by showing that for predetermined $\overline{\mathbf{H}}$ and $\overline{\mathbf{R}}$ there exists some time path $\boldsymbol{\Theta}$ for which a general equilibrium of the IEES exists (and which then obviously exhibits $\mathbf{H}=\overline{\mathbf{H}}$ and $\mathbf{R}=\overline{\mathbf{R}}$ ). To this end we maximize the consumer's present value utility subject to the constraints (1)-(14) and subject to the constraints $\bar{h}_{i}$ (all $i$ ) and $\bar{r}$. The corresponding Lagrangean reads

$$
\overline{\mathcal{L}}^{P}=\mathcal{L}^{P}+\sum_{i} \alpha_{\bar{h} i}\left(h_{i}-\bar{h}_{i}\right)+\alpha_{\bar{r}}(r-\bar{r})
$$

where $\mathcal{L}^{P}$ is given by (15). The first-order conditions are listed in column 1 of Table 1 where lines 2 and 9 a have to be replaced by

$$
\alpha_{v i}=\frac{\alpha_{y g}}{H_{y_{g}}^{i}}+\alpha_{z i}-\alpha_{\bar{h} i}
$$

and

$$
\dot{\rho}-\dot{\gamma}=(\rho-\gamma)\left(\delta-R_{r}\right)-\alpha_{r}-\alpha_{\bar{r}}-\sum_{k} U_{q_{c k}} Q_{r}^{k}+\alpha_{s} .
$$

The first-order conditions characterize a Pareto efficient allocation constrained by $h_{i}=\bar{h}_{i}$ and $r=\bar{r}$. The following lemma can be proven along the same lines as Proposition 1.

\section{Lemma 1.}

If the function $U$ is quasi-concave and the functions $B^{i}$ (all $i$ ), $F, H^{i}$ (all $i$ ), $Q^{k}$ (all $k$ ), $R$ are concave, a solution $\left(\boldsymbol{\alpha}, \boldsymbol{\beta}, \boldsymbol{\gamma}, \boldsymbol{\rho}, \boldsymbol{A}^{m}, \boldsymbol{A}^{y}\right)$ to the Hamiltonian (15') exists.

Next, setting $p_{h i}=\alpha_{v i}($ all $i), p_{r}=\alpha_{r}, p_{y g}=\alpha_{y g}($ all $g), \pi_{z i}=\alpha_{z i}>0$ (all i), $\pi_{s}=\alpha_{s}$, $\theta_{r}=\pi_{s}-\sum_{k} \frac{p_{y g} U_{q_{c k}} Q_{r}^{k}}{U_{y_{c g}}}-\alpha_{\bar{r}}$ (any $g$ ), $\theta_{h i}=\pi_{z i}-\alpha_{\bar{h} i}$ (all i) renders identical the equations 
in lines 1-11 of column 1 (where 2 and 9a are replaced by 2' and 9a') of Table 1 and the equations in lines 1-11 of column 2 of Table 1 . The matching of these columns together with Lemma 1 proves that for predetermined $(\overline{\mathbf{H}}, \overline{\mathbf{R}})$ there is a set of time paths of taxes, say $\mathrm{M}(\overline{\mathbf{H}}, \overline{\mathbf{R}})$, such that for any $\boldsymbol{\Theta} \in \mathrm{M}(\overline{\mathbf{H}}, \overline{\mathbf{R}})$ there exists a general equilibrium of the IEES with open access habitat characterized by $\mathbf{H}=\overline{\mathbf{H}}$ and $\mathbf{R}=\overline{\mathbf{R}}$.

Define $\Omega:=\{\boldsymbol{\Theta} \mid \boldsymbol{\Theta} \in \mathrm{M}(\overline{\mathbf{H}}, \overline{\mathbf{R}})$ for all $\overline{\mathbf{H}} \geq 0, \overline{\mathbf{R}} \geq 0\}$. We clearly showed that $\Omega$ is nonempty and that associated to every $\Theta \in \Omega$ there is an equilibrium of the IEES. In fact, there is no equilibrium of the IEES for predetermined $\Theta$, if $\Theta \notin \Omega$. To validate that claim suppose the contrary, i.e. suppose that there is some $\tilde{\Theta} \notin \Omega$ supporting an equilibrium. The associated equilibrium allocation then entails some specific time path of harvesting and land conversion, say $\tilde{\mathbf{H}}$ and $\tilde{\mathbf{R}}$. But we showed above that for $(\tilde{\mathbf{H}}, \tilde{\mathbf{R}})$ there exists a set of tax paths, $\mathrm{M}(\tilde{\mathbf{H}}, \tilde{\mathbf{R}}) \neq \emptyset$ which necessarily contains $\tilde{\boldsymbol{\Theta}}$ as an element. Hence $\tilde{\boldsymbol{\Theta}} \in \Omega$. This contradiction proves that there exists a general equilibrium for the IEES with open access habitat for predetermined $\Theta$, if and only if $\Theta \in \Omega$.

The proof of Propositions 4 and 5, respectively, is analogous to the proof of Propositions 2 and 3, respectively, and is therefore omitted.

\section{Proof of Proposition 6:}

Equations (26), (27) and (29) follow from the equations in lines 2 and 9 (column general equilibrium) of Table 1 taking into account $\theta_{h i}$ and $\theta_{r}$ from Proposition 2.

Equation (28) is proven as follows: From line 10 in Table 1:

$$
\beta_{i}^{*}=\frac{1}{\delta}\left(\sum_{k} \frac{p_{y g}^{*} U_{q_{c k}} Q_{n_{i}}^{k}}{U_{y_{c g}}}+\frac{p_{y g}^{*} H_{n_{i}}^{i}}{H_{y_{i g}}^{i}}-\pi_{r}^{*} r_{i}-\sum_{j} \pi_{z j}^{*} z_{i j}\right) .
$$

Making use of the budget constraint defined in (16):

$$
\beta_{i}^{*}=\frac{1}{\delta}\left(\sum_{k} \frac{p_{y g}^{*} U_{q_{c k}} Q_{n_{i}}^{k}}{U_{y_{c g}}}+\frac{p_{y g}^{*} H_{n_{i}}^{i}}{H_{y_{i g}}^{i}}-\pi_{r}^{*} \omega_{i}-\frac{\pi_{z i}^{*} h_{i}}{n_{i}}\right) .
$$

Invoking (26) we obtain

$$
\frac{p_{y g}^{*} H_{n_{i}}^{i}}{H_{y_{i g}}^{i}}=\left(p_{h i}^{*}-\pi_{z i}^{*}\right) H_{n_{i}}^{i}=\frac{\left(p_{h i}^{*}-\pi_{z i}^{*}\right) h_{i}}{n_{i}} \cdot \frac{n_{i} H_{n_{i}}^{i}}{h_{i}} .
$$

Equations (30) and (31) follow from the first order conditions

$$
\begin{aligned}
& \dot{\rho}=\rho \delta-\rho R_{r}+\gamma R_{r}-\alpha_{r}-\sum_{k} \alpha_{q k} Q_{r}^{k}, \\
& \dot{\gamma}=\gamma \delta-\alpha_{s}
\end{aligned}
$$


of (15). Setting $\dot{\rho}=\dot{\gamma}=0$ and using the information of Proposition 2 yields the desired equations. 